\title{
TITLE:
}

\section{Competition between interchain and intrachain phase segregation}

AUTHOR(S):

Iwaki, T; Yoshikawa, K

\section{CITATION:}

Iwaki, T ...[et al]. Competition between interchain and intrachain phase segregation. Physical Review E 2003, 68(3): 031902.

ISSUE DATE:

2003-09

URL:

http://hdl.handle.net/2433/49846

RIGHT:

Copyright 2003 American Physical Society 
PHYSICAL REVIEW E 68, 031902 (2003)

\title{
Competition between interchain and intrachain phase segregation
}

\author{
T. Iwaki and K. Yoshikawa \\ Department of Physics, Graduate School of Science, Kyoto University, Kyoto 606-8502, Japan
}

(Received 5 February 2003; published 8 September 2003)

\begin{abstract}
Single-molecule observations of giant DNA have clarified that individual molecules undergo a marked discrete transition between an elongated coil state and a compact globule state. There is a relatively wide region of coexistence between the coil and the globule states, i.e., interchain phase segregation, with a change in intensive variables such as the concentration of the condensing agent, salt concentration, temperature. Very recently, the coexistence of coil and globule conformations within a single long DNA chain, i.e., intrachain phase segregation, has been reported under certain experimental conditions. In this study, we investigated general conditions for intrachain phase segregation in a single polyelectrolyte molecule, based on a simple statistical model. We consider the contribution of condensed counterions and the interaction energy of a charged coiled region. Intrachain phase segregation is stable with regard to free energy within a suitable parameter region. Our results suggest that intrachain phase segregation occurs when the electrostatic screening effect by the salt solution is negligible or when the screening effect is large and there is attractive interaction between polyelectrolyte segments.
\end{abstract}

DOI: 10.1103/PhysRevE.68.031902

PACS number(s): 87.15.By, 64.75.+g, 47.20.Hw, 42.70.Jk

\section{INTRODUCTION}

A coil-globule transition is found in monomolecular linear polymeric chains, where the "coil" is an elongated, fluctuating state and the "globule" is a compact folded state. This biphasic nature of the conformation of linear macromolecules has been extensively studied both theoretically [1-6] and experimentally [7-12]. In general, polymer solutions show phase separation between a dense layer and a dilute layer $[1,13,14]$ as a multichain process, which has been studied within the framework of the mean-field theory by Flory [1]. The application of this framework to individual long polymeric chains led to the expectation that interchain phase segregation between the coil and the globule phases would appear in very dilute polymer solutions where monomolecular dispersion can be realized [2-4]. However, there were two serious problems in the application of the mean-field theory to individual molecular chains, i.e., the surface energy and the effect of fluctuations. Based on the surface energy from the self-consistent field theory for single polymers $[5,6]$, it has been suggested that a stiff long polymer undergoes a first-order coil-globule transition, i.e., interchain phase segregation, although most experimental studies did not support the appearance of bimodality in single-chain conformations [15]. Such bimodality has been demonstrated experimentally [8] for a stiff long polyelectrolyte, i.e., giant DNA, by means of monomolecular observation.

On the other hand, there still remains the problem of fluctuation, or whether a coil part and a globule part can coexist within a single polymer chain. Recent experiments on DNA [16-19] and synthetic polyelectrolytes [20] have indicated that such intrachain phase segregation does indeed exist. The densities of the elongated part and the compact part in the segregated chain were essentially equal to those of a simple DNA coil and a simple DNA globule, respectively. This suggests that each polymer segment in a single segregated chain assumes either of the phases seen in interchain phase segregation. Thus, the problem here is not "What phase appears?" but rather "How can two phases appear?" Possible routes in coil-globule transition are shown schematically in Fig. 1.

Simulation studies on polyelectrolytes [21-24] have shown the formation of a similar necklace structure. The necklace structure has been discussed in terms of the sizedependent stability of the globule part. When we model a globule as a uniformly charged sphere, the repulsive electrostatic self-energy of the globule is equal to the volume of a sphere raised to the power $5 / 3$. As the globule increases in size, this repulsive energy overwhelms the other extensive attractive contributions. This scenario is called Rayleigh instability, and leads to a cascade of abrupt transitions between an $N$-bead necklace and an $(N+1)$-bead necklace [21]. It is to be noted that some theoretical studies discussed "Rayleigh instability" on a necklace by neglecting the effect of counte-

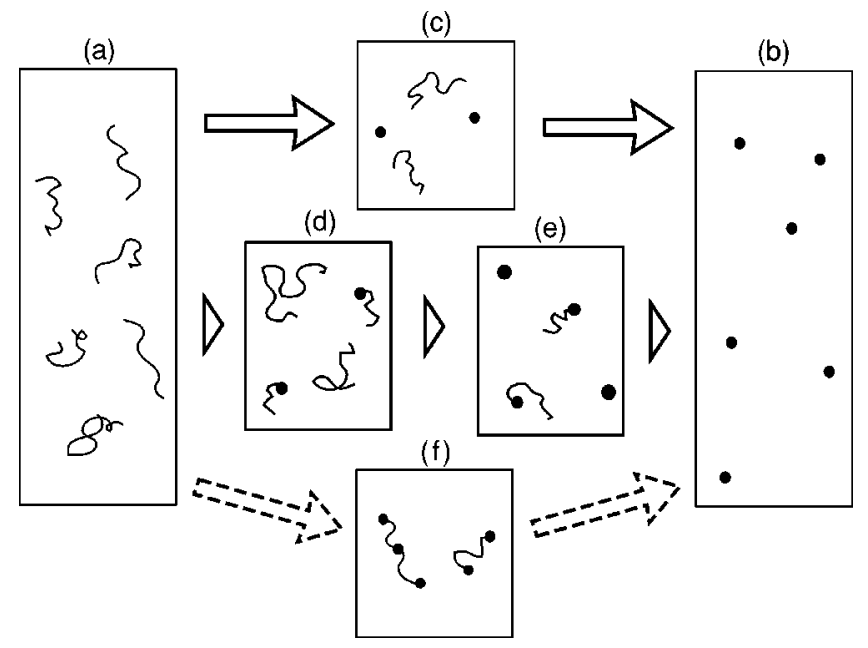

FIG. 1. Schematic diagram of possible pathways in the coilglobule transition. (a) Full coil. (b) Full globule. (c) Coexistence of the full coil and full globule (interchain phase segregation). (d) Coexistence of the full coil and intrachain phase segregation. (e) Coexistence of the full globule and intrachain phase segregation. (f) Intrachain phase segregation in some more complicated cases. 
rions. However, if we consider polyelectrolytes in an aqueous environment, preservation of the negative charge upon the folding on collapsing transition is the situation hardly generated in experiments in the real world. In fact, a previous experimental observation of intrachain phase segregation [17] suggested that the unfolded and folded parts in a segregated chain show a large difference in charge density. If the internal charge of a globule part is fully neutralized by counterions, Rayleigh instability cannot occur. Unfortunately, the absolute charge on the folded part in a segregated structure has not yet been measured experimentally. However, the enormous energy of repulsive electrostatic interaction for a large globule should promote charge neutralization by counterions. For example, an actual experiment involving the electrophoresis of T4 DNA suggested complete charge neutralization in the volume part of a tightly packed DNA molecule [25].

In the present work, to determine the intrinsic properties of intrachain segregation, we performed a theoretical study of the possibility of intrachain phase segregation, even in the absence of Rayleigh instability. For this purpose, we adopt an extreme situation in which the charge of a folded part is assumed to be neutral. Two contributions to the free energy of a single polyelectrolyte chain are considered: the free energy of the condensed counterion configuration and the intersegment interaction energy in a coil.

\section{FREE ENERGY ON A POLYELECTROLYTE CHAIN}

We consider a long stiff polyelectrolyte chain. The present model can be outlined as follows. We consider intrachain phase segregation as a conformation consisting of one coil part and one globule part. Bimodality in local chain conformation is assumed for each polyelectrolyte segment. Instead of using concrete order parameters, we designate directly whether each segment assumes the coil phase or the globule phase, where the globule phase is modeled as a compact conformation with constant density where the whole charge is fully neutralized. The coil phase is approximated with a Gaussian chain and its corresponding intersegment interaction energy. Intersegment interaction in a real system deforms the polyelectrolyte conformation relative to a Gaussian chain. This deformation is significant in the globule phase but not too extensive in the coil phase. The contour length of a polyelectrolyte chain is denoted as $L_{0} . L_{c} / L_{0}$ is the fraction of the coil part and $L_{g} / L_{0}$ is that of the globule part. In the present paper we focus on the nonlinearity of free energy. We are interested in determining the route via which the system undergoes the coil-globule transition (Fig. 1). A linear term for free energy to $L_{g} / L_{0}\left(=1-L_{c} / L_{0}\right)$ determines the stage along a given route a polyelectrolyte chain is in (Fig. 2 ). Thus, two additional terms are considered as nonlinear contributions to the free energy of a single polyelectrolyte chain. One is the contribution of the condensed counterion configuration and is calculated from the Ising model. The other is the intersegment interaction energy in the coil part.

We have already fully discussed the mechanism of the coil-globule transition [8,26-28]. For simplicity, we ignore surface effects and osmotic pressure. Both contributions ba-

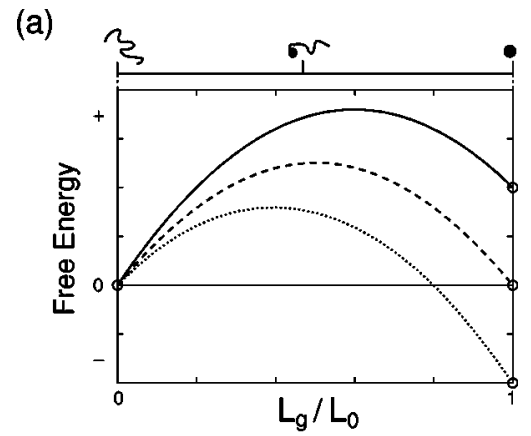

(b)

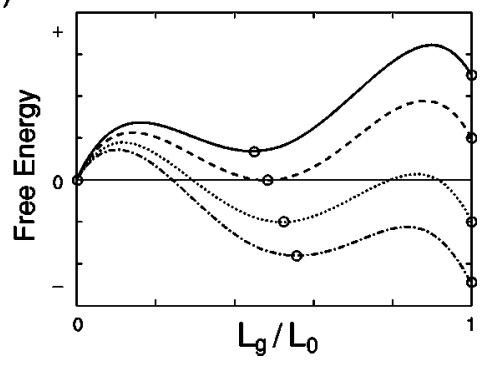

FIG. 2. Free energy vs the polymer conformation. The nonlinear term of free energy is fixed. The coil-globule transition is driven by changing the linear term. Circles indicate local minima. (a) This case represents the route $\mathbf{a} \rightarrow \mathbf{c} \rightarrow \mathbf{b}$ in Fig. 1. The solid line corresponds to $\mathbf{a}$, the dashed line corresponds to $\mathbf{c}$, and the dotted line corresponds to b. (b) This case represents the route $\mathbf{a} \rightarrow \mathbf{d} \rightarrow \mathbf{e} \rightarrow \mathbf{b}$ in Fig. 1. The solid line corresponds to a, the dashed line corresponds to $\mathbf{d}$, the dotted line corresponds to $\mathbf{e}$, and the dot-dash line corresponds to $\mathbf{b}$.

sically stabilize interchain phase segregation. The surface energy is essential particularly for the globule where singularity at the surface is notable. The surface energy has the scaling $V^{2 / 3}$ where $V$ is the volume of the phase structure. From the morphology, it is natural to expect that the surface energy of a globule is positive. The osmotic pressure of water originates from the mixing entropy. This contribution is reduced into a local repulsive virial-type interaction. For the globule, this contribution would be almost linear with respect to the volume. For the coil, such contribution leads to interchain phase segregation as shown later. The osmotic pressure of counterions is considered mainly between the salty bulk and counterion-condensed volume on the polyelectrolyte. This contribution scales with the mass volume of the coil and globule, respectively. In addition, we have not taken into account the interaction between the coiled region and the globule, which is associated with the morphology of intrachain phase segregation rather than the stabilization of intrachain phase segregation.

First, we derive the free energy of condensing counterions. In strongly charged polyelectrolyte systems, counterions condense strongly on a polyelectrolyte rod and neutralize its charge to a large extent $[29,30]$. We calculate the contribution of a condensed counterion configuration with a onedimensional Ising model. We try to remain consistent with the counterion condensation theory [30] through the selfconsistent determination of the mean field, which is discussed later (Sec. III). Usual experimental systems that show 
the coil-globule transition consist of counterions with various valences. Normally, different kinds of counterions compete with each other for binding sites [30,31]. Monovarent counterions have been reported to experimentally induce the retardation of polyelectrolyte precipitation by multivalent counterions [32-34]. For simplicity, we only consider the condensation of a single kind of counterion which induces the coil-globule transition. A typical Hamiltonian for the Ising model can be described as follows:

$$
-\beta \mathcal{H}=J \sum \sigma_{i} \sigma_{i+1}+H \sum \sigma_{i},
$$

where $\beta$ is the inverse of the temperature, $J$ is the neighboring interaction, and $H$ is the external field. Each spin indicates whether or not a counterion is bound at each site. In real systems, there are distant-pair interactions produced by long-range electrostatic interaction. In a globule structure, contact between polymer segments enables nonlocal interactions. These contributions are not considered explicitly in the main body of the paper. The interactions can be determined self-consistently within the framework of the mean-field theory to incorporate the above contributions, which will be discussed later. In this case, $H$ and $J$ should be specific to the coil and globule phases, respectively, as $J_{c}, J_{g}, H_{c}$, and $H_{g}$. We initially regard all of these coefficients as parameters.

The partition function of condensed counterions is described with Onsager's transfer matrix [35]:

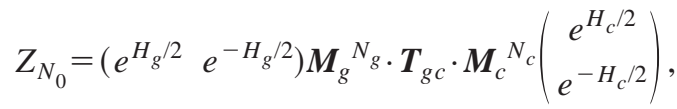

where

$$
\begin{gathered}
M_{g}=\left(\begin{array}{cc}
e^{J_{g}+H_{g}} & e^{-J_{g}} \\
e^{-J_{g}} & e^{J g-H_{g}}
\end{array}\right), \\
M_{c}=\left(\begin{array}{cc}
e^{J_{c}+H_{c}} & e^{-J_{c}} \\
e^{-J_{c}} & e^{J_{c}-H_{c}}
\end{array}\right), \\
T_{g c}=\left(\begin{array}{cc}
e^{J+\left(H_{g}+H_{c}\right) / 2} & e^{-J+\left(H_{g}-H_{c}\right) / 2} \\
e^{-J-\left(H_{g}-H_{c}\right) / 2} & e^{J-\left(H_{g}+H_{c}\right) / 2}
\end{array}\right) .
\end{gathered}
$$

$N_{g}$ is the number of adsorption sites in the globule domain, $N_{c}$ is the number of such sites in the coil domain, and $J$ is the neighboring interaction at the interface between the coil and the globule states. $N_{g}$ and $N_{c}$ are equivalent to $L_{g}$ and $L_{c}$, respectively. The partition function is calculated as

$$
Z_{N_{0}}=\left(A_{g} B_{g}\right)\left(\begin{array}{cc}
e^{J} & e^{-J} \\
e^{-J} & e^{J}
\end{array}\right)\left(\begin{array}{l}
A_{c} \\
B_{c}
\end{array}\right),
$$

where, by taking $i=g$ or $c$,

$$
A_{i}=\left(e^{H_{i}} X_{i}+e^{H_{i}} Y_{i}+Z_{i}\right) \lambda_{i+}^{N_{i}}+\left(e^{H_{i}} X_{i}-e^{H_{i}} Y_{i}-Z_{i}\right) \lambda_{i-}^{N_{i}},
$$

$$
\begin{gathered}
B_{i}=\left(e^{-H_{i}} X_{i}-e^{-H_{i}} Y_{i}+Z_{i}\right) \lambda_{i+}^{N_{i}} \\
+\left(e^{-H_{i}} X_{i}+e^{-H_{i}} Y_{i}-Z_{i}\right) \lambda_{i-}^{N_{i}}, \\
X_{i}=\sqrt{e^{2 J_{i}} \sinh ^{2}\left(H_{i}\right)+e^{-2 J_{i}},} \\
Y_{i}=e^{J_{i}} \sinh \left(H_{i}\right), \\
Z_{i}=e^{-J_{i}}, \\
\lambda_{i \pm}=e^{J_{i}} \cosh \left(H_{i}\right) \pm X_{i},
\end{gathered}
$$

$\lambda_{g \pm}$ and $\lambda_{c \pm}$ are the characteristic roots of each transfer matrix. Within the framework of the mean-field theory, all coefficients $H_{i}$ and $J_{i}$ represent macroscopic properties of the coil and globule. On the other hand, the interface does not form a macroscopic structure. Thus, the neighboring interaction at the interface $J$ is not derived from the mean-field theory. We consider here that $J$ equals 0 . This corresponds to division of the whole system into two energetically independent parts. Finally, the dimensionless Ising free energy $F_{I}$ $=-\ln Z_{N_{0}}$ takes the form

$$
\begin{aligned}
& F_{I}=-\ln \left(1+b_{g} e^{-a_{g}^{-1} L_{g} / L_{0}}\right)-\ln \left(1+b_{c} e^{-a_{c}^{-1} L_{c} / L_{0}}\right) \\
& +\left[\ln \left(1+b_{g} e^{-a_{g}^{-1}}\right)+\ln \left(1+b_{c}\right)\right] \frac{L_{g}}{L_{0}} \\
& +\left[\ln \left(1+b_{g}\right)+\ln \left(1+b_{c} e^{-a_{c}^{-1}}\right)\right] \frac{L_{c}}{L_{0}},
\end{aligned}
$$

where

$$
\begin{gathered}
a_{i}=\left[N_{0} \ln \left(\lambda_{i+} / \lambda_{i-}\right)\right]^{-1}, \\
b_{i}=\frac{\cosh \left(H_{i}\right) X_{i}-\sinh \left(H_{i}\right) Y_{i}-Z_{i}}{\cosh \left(H_{i}\right) X_{i}+\sinh \left(H_{i}\right) Y_{i}+Z_{i}} .
\end{gathered}
$$

The third and fourth terms in $F_{I}$ are introduced to graphically describe the nonlinear dependency, so that $F_{I}$ takes the value 0 on both ends of the intrachain phase segregation, i.e., $L_{g} / L_{0}=0$ and $L_{g} / L_{0}=1 . F_{I}$ gives the plateaulike potential barrier between the full-coil state and the full-globule state, which makes intrachain phase segregation unstable. The parameter $a_{i}$ gives the slope width, which determines the steepness of the convex curve of $F_{I}$. We consider the system where $\lambda_{i+} / \lambda_{i-} \approx 1$, i.e., the convex curve is relatively gradual. The parameter $b_{i}$ determines the height of the convex curve.

We now address the scaling of the intersegment interaction energy in the coil part. A polymer conformation in the coil phase is modeled as a Gaussian chain as described above, while the globule has a fully electrostatically neutralized [25] structure with uniform density. We consider scaling only for the coil part because intersegment interaction in the globule, such as counterion-mediated attraction [29,36-40], should only produce the linear term of interaction energy for a relatively large globule. We start our argument from the 
well-known relation of the mean squared distance of the endto-end vector for a Gaussian chain $[13,14]$ :

$$
\left\langle R^{2}\right\rangle=2 L_{c} l_{p}
$$

where $l_{p}$ is the persistence length. If we assume that a coil is spherical, its radius $R_{0}$ is

$$
R_{0}=\sqrt{\left\langle R^{2}\right\rangle} .
$$

The polyelectrolyte solution feels some degree of electrostatic screening by the salt. When this screening effect is relatively negligible, the whole dimensionless electrostatic energy of the coil (electrostatic energy divided by the thermal energy) $E_{c, 0}$ follows

$$
\frac{\partial E_{c, 0}}{\partial L_{c}} \sim L_{c} / R_{0} \sim L_{c}{ }^{1 / 2} .
$$

This scaling is valid when the Debye length is larger than the coil part. Equation (15) leads to

$$
E_{c, 0}=E_{0}\left(L_{c} / L_{0}\right)^{3 / 2}-E_{0}\left(L_{c} / L_{0}\right),
$$

where $E_{0}$ is a dimensionless constant parameter. The second term of $E_{c, 0}$ is introduced to graphically describe the nonlinear dependency. There could be some short-range interaction, as represented by the virial formalism. However, the long-range electrostatic repulsive interaction dominates the effective intersegment interaction felt by the coil under conditions of negligible screening. The electrostatic energy has a positive second derivative against the coil fraction $L_{c} / L_{0}$, resulting in intrachain phase segregation. When the solution has a strong screening effect, the whole interaction energy undergoes serious deformation. Simple Debye-Hückel-type screening could make this very complicated. We consider here that the interaction energy in such a case does not depend on the whole geometry of the coil structure, but rather only on the local polymer concentration. The interaction is then represented by the virial formalism. We consider up to the second virial term for the intersegment interaction of a coil. The dimensionless interaction energy in this case, $E_{c, s}$, is

$$
\begin{gathered}
\frac{\partial E_{c, s}}{\partial L_{c}} \sim B \Phi_{p} \sim L_{c}^{-1 / 2}, \\
E_{c, s}=E_{0}^{\prime}\left(L_{c} / L_{0}\right)^{1 / 2}-E_{0}^{\prime}\left(L_{c} / L_{0}\right),
\end{gathered}
$$

where $B$ is the second virial coefficient, $E_{0}^{\prime}$ is a dimensionless constant parameter, and $\Phi_{p}$ is the local concentration at the periphery of the coil part:

$$
\Phi_{p}=\frac{\partial L_{c}}{\partial V_{R_{0}}}=\frac{1}{2 \pi L_{c}^{1 / 2}\left(2 l_{p}\right)^{3 / 2}}
$$

As noted above, the second term of $E_{c, s}$ is introduced to graphically describe nonlinear dependency. The second virial coefficient can be positive or negative according to the environment. If the effective intersegment interaction in the coil

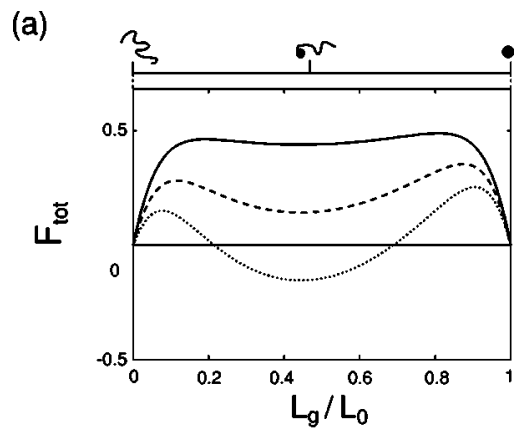

(b)

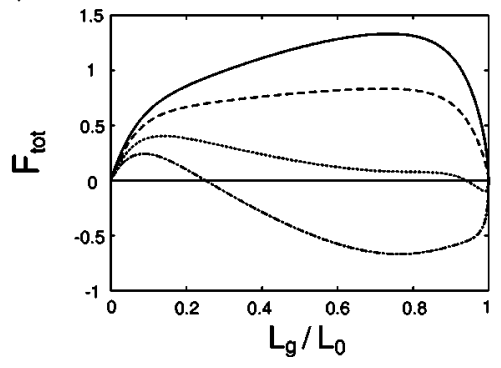

FIG. 3. Dimensionless free energy $F_{\text {tot }}$ vs the polyelectrolyte conformation. The parameters in the contribution of condensed counterions have the following values: dumping factor $a_{g}=0.05$, $a_{c}=0.05 ;$ and the magnitude of the contribution, $b_{g}=0.8, b_{c}$ $=0.8$. (a) Negligibly weak screening. Solid line, scaling parameter of intersegment interaction, $E_{0}=1$; dashed line, $E_{0}=3$; and dotted line, $E_{0}=5$. (b) Strong screening. Solid line, scaling parameter, $E_{0}^{\prime}=3$; dashed line, $E_{0}^{\prime}=1$, dotted line, $E_{0}^{\prime}=-2$, and dot-dash line, $E_{0}^{\prime}=-5$.

phase is attractive, the profile of interaction energy is convex downward and intrachain phase segregation is stabilized.

Figure 3 shows a profile of the total dimensionless free energy $F_{\text {tot }}$,

$$
F_{t o t}=F_{I}+E_{c, i},
$$

for the two cases described above. Three different conformational states are formed, separated by potential barriers, in the case of a negligible screening effect. Intrachain phase segregation is realized when the mid local minimum is below the horizontal line. When the mid local minimum is above the horizontal line, intrachain phase segregation is metastable. When the effective intersegment interaction in the coil is attractive, as in a poor solvent, intrachain phase segregation can be generated in a strong screening environment, where no potential barrier appears between the full-globule state and intrachain phase segregation, in contrast to the case with weak screening. However, this lack of a potential barrier in the case of strong screening is considered to be artificial, and is attributed to the unrealistic divergence of the coil density under the limit of $L_{c} \rightarrow 0$. When $L_{c}$ is not too large compared to the persistence length, the actual coil conformation is much different from that of a Gaussian chain.

\section{DISCUSSION}

We derived the free energy of the condensed counterion configuration and the inter-segment interaction energy of the 
coil part to discuss the nonlinearity of the free-energy profile with respect to the polyelectrolite conformation. Intrachain phase segregation was stabilized (a) with a negligibly lowsalt solution, where repulsive electrostatic interaction dominates intersegment interaction and (b) when long-range electrostatic interaction decays very rapidly under a highly screening environment and the second virial coefficient that describes the system is effectively attractive.

Experiments have shown that a system of polyelectrolytes with multivalent counterions experiences intrachain phase segregation under a fairly high salt concentration [16], while a low-salt concentration does not induce intrachain phase segregation. Our results suggest that the second virial coefficient representing intersegment interaction in the coil phase should be attractive under such experimental conditions. The second virial term is a particular kind of average between a screened repulsive electrostatic interaction and a very shortrange attractive interaction $[29,36-40]$ caused by the correlation of condensed counterions. For low-salt conditions, it is natural to consider that the contribution to the second virial term in the coil is dominated by electrostatic repulsive interaction. The observed invariance of the coil size during interchain phase segregation with multivalent counterions [41-44] is associated with such a repulsive second virial coefficient. In contrast, when the salt concentration increases, the repulsive electrostatic interaction decays much faster. The contribution of short-range attractive interaction relatively increases. The value of the second virial coefficient should change from positive to negative with a salt increase, resulting in intrachain phase segregation. The alternative scenario of intrachain phase segregation, i.e., with negligible salt, is difficult to realize under usual experimental conditions. Equation (15) requires the relations $L_{c}>l_{p}$ and $\lambda_{D}$ $>R_{0}$, where $\lambda_{D}$ is the Debye length. These relations together become

$$
l_{p}<L_{c}<\frac{\lambda_{D}^{2}}{2 l_{p}} .
$$

Taking into account that the persistence length is the sum of the intrinsic persistence length and the electrostatic contribution, i.e., $l_{p}=l_{p, o}+\left(\lambda_{B} / 4\right)\left(\lambda_{D} / b \xi\right)^{2}$, Eq. (21) has a solution under the following condition:

$$
l_{p, o}<\frac{b^{2} \xi^{2}}{2 \lambda_{B}}=\frac{\lambda_{B}}{2} .
$$

$\lambda_{B}$ is the Bjerrum length, $b$ is the axial charge spacing of polyelectrolyte, and the factor $\xi=\lambda_{B} / b$ is derived from Manning's counterion condensation. Equation (22) indicates that the scaling equation (15) is valid under the limited conditions of an intrinsically flexible polyelectrolyte. This is not the case for DNA.

We now discuss the parameter $b_{i}$, which appears in the free energy of the condensed counterion configuration, $F_{I}$. The dumping factor $a_{i}$ is not critical to our argument. The parameter $b_{i}$ determines the height of the plateaulike potential barrier. In principle, the magnitude of $F_{I}, b_{i}$, has a value between 0 and 1 . We briefly discuss here how to estimate the value of $b_{i}$ while taking into account the counterion valency. First, there is the relation between the interaction coefficient $J_{i}$ and the external field $H_{i}$. The following relation is recognized from the Hamiltonian [Eq. (1)]:

$$
N_{i}\langle\sigma\rangle_{i}=\frac{\partial \ln Z_{N_{0}}}{\partial H_{i}} .
$$

We transform this relation into that for the macroscopically expected mean spin:

$$
\langle\sigma\rangle_{i}=\frac{\partial \ln \lambda_{i+}}{\partial H_{i}}=\frac{x}{\sqrt{x^{2}+s^{2}}}=\sin \theta,
$$

where $x=\sinh H_{i}$ and $s=e^{-2 J_{i}}$. The last equality is obtained from the transformation $x=s \tan \theta(-\pi / 2 \geqslant \theta \geqslant \pi / 2)$. Equation (12) can be rewritten as

$$
b_{i}=\frac{\sqrt{1+x^{2}} \sqrt{x^{2}+s^{2}}-x^{2}-s}{\sqrt{1+x^{2}} \sqrt{x^{2}+s^{2}}+x^{2}+s} .
$$

For a strongly charged polyelectrolyte such as DNA, $s$ is much larger than unity. If $|\tan \theta| \gg 1 / \sqrt{s}$,

$$
b_{i} \simeq \frac{1-|\sin \theta|}{1+|\sin \theta|} .
$$

First, we consider a globule, in which the whole charge is fully neutralized. One adsorption site corresponds to one charged group of the polyelectrolyte (- $\mathrm{PO}_{4}^{-}$- in DNA). This leads to the relation

$$
\langle\sigma\rangle_{g}=\frac{2}{z_{c}}-1
$$

where $z_{c}$ is the valence of the counterion. In the case of monovalent counterions, $\sin \theta=1$ and $b_{g}=0$. Divalent counterions give $x=s \tan \theta=0$ and $b_{g}=0$. In these two cases, there is no nonlinearity in the free energy of the globular domain. For $z_{c}=3, b_{g}=1 / 2$; for $z_{c}=4, b_{g}=1 / 3$, and so on. Next, we consider a coil, where the whole charge follows the counterion condensation theory $[29,30]$. The mean spin of the coil domain is

$$
\langle\sigma\rangle_{c}=2\left(\frac{1}{z_{c}}-\frac{1}{z_{c}^{2} \xi}\right)-1 .
$$

In DNA, $\xi \gg 1$. When $z_{c}=1$, the magnitude of $F_{I}, b_{c}$, is $1 /(\xi-1) \ll 1$. This is almost the same as in a globule. However, for $z_{c}=2, b_{c}$ is $(2 \xi-1) /(2 \xi+1) \simeq 1$, since $|\tan \theta|$ $\simeq 1 /(2 \xi) \gg 1 / \sqrt{s}$ under the assumption that the neighboring interaction $J_{c}$ is repulsive and of the order of the magnitude of the electrostatic interaction between neighboring counterions, $z_{c}^{2} \xi$. The value of $b_{i}$ for another valence shows almost the same property as in a globule.

Let us summarize these considerations. The valence of the counterions influences the height of the potential barrier. For a coil, a valence number greater than 2 induces a nonlinear 
profile, while a valence number greater than 3 does so for a globule. If the valence number increases, the potential barrier decreases. This means that a larger valence makes fluctuations more frequent, and sometimes realizes a continuous transition from a full-coil to a full-globule state through intrachain phase segregation. In reality, a large valence counterion may be quite large and is considered to be bound to several sites rather than just one site. In a more realistic case, the system contains two or more kinds of counterions. A more delicate treatment is necessary to discuss such problems.

We considered the salt effect with a much simpler approximation. In fact, the effect of salt ions is a very delicate problem [45] and the mean-field approach often becomes dangerous. For example, the possibility of charge inversion due to counterions has been reported $[46,47]$. Ion-ion correlation in the electrolyte system is also essential for such phenomena as ionic crystallization of the polyelectrolyte and short-range attractive interaction between polyelectrolyte rods $[29,36-40]$.

Finally, we discuss the pearl necklace in Fig. 1(f). This type of intrachain phase segregation has been found under some experimental studies [17-20]. Let us express these necklaces as $g-c-g, g-c-g-c-g$, and so on, where $c$ and $g$ denote a coil and a globule, respectively. Unfortunately, these necklaces are not stable in the present model. In the present model, a $g-c$ necklace is always more stable than $g-c-g, c-g-c$ is more stable than $g-c-g-c-g, c-g-c-g-c$ is more stable than $g-c-g-c-g-c-g$, and so on, since they only include a term to limit the size of a coil. To stabilize a $g$-c-g-type necklace, a contribution from limiting the size of a globule is required. One possibility is the presence of residual charges inside a globule. If there are residual charges, Rayleigh instability [21] could lead to $g-c-g$-type intrachain phase segregation. However, the problem of residual charges inside a globule is very delicate. This is left as a future problem. It may be possible to explain intrachain phase segregation by a combination of the present model and the effect of residual charges.

\section{CONCLUSION}

We investigated the nonlinearity of the free energy of a single polyelectrolyte chain with respect to its conformation (Figs. 2 and 3). This nonlinearity has a significant effect near the transition point where the linear term of free energy becomes very small. It has been shown that such a nonlinear effect can explain the existence of intrachain phase segregation. Two different cases were considered to stabilize intrachain phase segregation. In one case, the solution has a negligible salt concentration compared to the size of the segregated structure. In the other case, under high-salt conditions, the second virial coefficient for the coil phase becomes negative, i.e., the effective equalized intersegment interaction in the coil is attractive. The latter case is more closely associated with real experiments. In the present work, we only considered intrachain phase segregation consisting of one globule part and one coil part. However, more complicated intrachain phase segregation has been observed in actual experiments. Further research is needed to elucidate this problem.
[1] P.J. Flory, Principles of Polymer Chemistry (Cornell University Press, Ithaca, NY, 1952).

[2] Y.E. Eisner, Polym. Sci. U.S.S.R. 11, 409 (1969).

[3] P.G. de Gennes, J. Phys. (France) Lett. 36, 55 (1975).

[4] C.B. Post and B.H. Zimm, Biopolymers 21, 2123 (1982).

[5] I.M. Lifshitz, Sov. Phys. JETP 28, 1280 (1969).

[6] I.M. Lifshitz, A.Y. Grosberg, and A.R. Khokhlov, Rev. Mod. Phys. 50, 683 (1978).

[7] L.S. Lerman, Proc. Natl. Acad. Sci. U.S.A. 68, 1886 (1971).

[8] V.V. Vasilevskaya, A.R. Khokhlov, Y. Matsuzawa, and K. Yoshikawa, J. Chem. Phys. 102, 6595 (1995).

[9] G. Swislow, S.T. Sun, I. Nishio, and T. Tanaka, Phys. Rev. Lett. 44, 796 (1980).

[10] I.H. Park, Q.W. Wand, and B. Chu, Macromolecules 20, 1965 (1987).

[11] I. Nishio, S.T. Sun, G. Swislow, and T. Tanaka, Nature (London) 281, 208 (1979).

[12] C. Wu and S. Zhou, Macromolecules 28, 8381 (1995).

[13] P.G. de Gennes, Scaling Concepts in Polymer Physics (Cornell University Press, Ithaca, NY, 1979).

[14] A.Y. Grosberg and A.R. Khokhlov, Statistical Physics of Macromolecules (American Institute of Physics, New York, 1994).

[15] J. Widom and R.L. Baldwin, J. Mol. Biol. 144, 431 (1980).

[16] S. Takagi, K. Tsumoto, and K. Yoshikawa, J. Chem. Phys. 114, 6942 (2001).
[17] M. Ueda and K. Yoshikawa, Phys. Rev. Lett. 77, 2133 (1996).

[18] K. Yoshikawa, Y. Yoshikawa, Y. Koyama, and T. Kanbe, J. Am. Chem. Soc. 119, 6473 (1997).

[19] Y. Yoshikawa, Y. Velichko, Y. Ichiba, and K. Yoshikawa, Eur. J. Biochem. 268, 2593 (2001).

[20] A. Kiriy, G. Gorodyska, S. Minko, W. Jaeger, P. Štěpánek, and M. Stamm, J. Am. Chem. Soc. 124, 13454 (2002).

[21] A.V. Dobrynin, M. Rubinstein, and S.P. Obukhov, Macromolecules 29, 2974 (1996).

[22] P. Chodanowski and S. Stoll, J. Chem. Phys. 111, 6069 (1999).

[23] A.V. Lyulin, B. Dünweg, O.V. Borisov, and A.A. Darinskii, Macromolecules 32, 3264 (1999).

[24] U. Micka and K. Kremer, Europhys. Lett. 49, 189 (2000).

[25] Y. Yamasaki, Y. Teramoto, and K. Yoshikawa, Biophys. J. 80, 2823 (2001).

[26] E.Yu. Kramarenko, A.R. Khokhlov, and K. Yoshikawa, Macromolecules 30, 3383 (1997).

[27] V.V. Vasilevskaya, A.R. Khokhlov, S. Kidoaki, and K. Yoshikawa, Biopolymers 41, 51 (1997).

[28] H. Noguchi and K. Yoshikawa, J. Chem. Phys. 109, 5070 (1998).

[29] F. Oosawa, Polyelectrolyte (Marcel Dekker, New York, 1971).

[30] G.S. Manning, Q. Rev. Biophys. 2, 179 (1978).

[31] I. Rouzina and V.A. Bloomfield, J. Phys. Chem. 100, 4292 (1996). 
[32] J. Pelta, F. Livolant, and J.-L. Sikorav, J. Biol. Chem. 271, 5656 (1996).

[33] E. Raspaud, M.O. de la Cruz, J.-L. Sikorav, and F. Livolant, Biophys. J. 74, 381 (1998).

[34] J.X. Tang and P.A. Janmay, J. Biol. Chem. 271, 8556 (1996).

[35] L. Onsager, Phys. Rev. 65, 117 (1944).

[36] I. Rouzina and V.A. Bloomfield, J. Phys. Chem. 100, 9977 (1996).

[37] B.I. Shklovskii, Phys. Rev. Lett. 82, 3268 (1999).

[38] B.Y. Ha and A.J. Liu, Phys. Rev. E 60, 803 (1999).

[39] J. Ray and G.S. Manning, Langmuir 10, 2450 (1994).

[40] F.J. Solis and M.O. de la Cruz, Phys. Rev. E 60, 4496 (1999).
[41] Y. Yamasaki and K. Yoshikawa, J. Am. Chem. Soc. 119, 10573 (1997).

[42] M. Takahashi, K. Yoshikawa, V.V. Vasilevskaya, and A.R. Khokhlov, J. Phys. Chem. B 101, 9396 (1997).

[43] S.M. Mel'nikov, V.G. Sergeyev, K. Yoshikawa, H. Takahashi, and I. Hatta, J. Chem. Phys. 107, 6917 (1997).

[44] N. Makita and K. Yoshikawa, FEBS Lett. 460, 333 (1999).

[45] M. Ullner and B. Jönsson, Macromolecules 29, 6645 (1996).

[46] T.T. Nguyen, A.Yu. Grosberg, and B.I. Shklovskii, J. Chem. Phys. 113, 1110 (2000).

[47] M. Tanaka, and A.Yu. Grosberg, J. Chem. Phys. 115, 567 (2001). 